\title{
A technique for rapid, reliable assessment of thermal-pain threshold in humans
}

\author{
KENNETH A. PERKINS, JAMES E. GROBE, J. RICHARD JENNINGS, \\ LEONARD H. EPSTEIN, and CELESTE ELASH \\ University of Pittsburgh School of Medicine, Pittsburgh, Pennsylvania
}

\begin{abstract}
A simple and reliable method of assessing pain threshold in humans may be useful in a number of research areas, such as in examining acute antinociceptive effects of drugs. We have developed a low-cost, computer-controlled method to reliably assess thermal-pain threshold using radiant heat focused from a slide projector and applied to a small area of skin. An electronic shutter is attached to the projector opening to provide precise onset and offset of heat. A computer keypad allows for subject response at the point of pain onset (i.e., threshold), with latency to threshold determined by computer timer. The details of the apparatus setup, subject preparation, and instructions to subjects are presented. The development of the method is described, and sources of unreliability are identified. Coefficients of variation $(C V s)$ are calculated to provide a measure of variability within subjects across trial exposures and across sessions. Results indicate that the method is reliable for determining pain threshold, especially within sessions, and can be employed with relatively minimal expense and subject preparation.
\end{abstract}

A simple, standardized method of assessing pain detection in humans may have a variety of applications in behavioral research, such as in basic, nonclinical studies on antinociceptive effects of drugs. A number of methods have been used to assess pain-detection threshold (i.e., onset of pain) and tolerance (i.e., endurance). Among the more common are cold pressor (immersing a hand in an ice bath; Pomerleau, Turk, \& Fertig, 1984), electric shock (Waller, Schalling, Levander, \& Edman, 1983), and controlled pressure on the skin (Seltzer, Friedman, Siegelaub, \& Collen, 1974). However, considerable adaptation or sensitization may occur over time to the pain produced by many of these methods (Beecher, 1959; Collier, Mehta, \& Spear, 1990; Handwerker, 1984), which could limit their usefulness in acute manipulations (such as drug administration) involving repeated pain assessments. Furthermore, many methods may be quite noxious, exacerbating variability in responding associated with individual differences in various psychological characteristics (Beecher, 1959; Chen, Dworkin, Haug, \& Gehrig, 1989).

Thermal stimulation of the skin may be a desirable method of determining pain threshold in humans because of the precision with which it can be manipulated and the general lack of adaptation it produces (Gracely, Lota, Walter, \& Dubner, 1988; Hardy, Wolff, \& Goodell, 1952; Kenshalo, Anton, \& Dubner, 1989), although adaptation to thermal-pain tolerance with very high intensity stimuli

This research was supported by Grant R01-DA05807 from the National Institute on Drug Abuse. The authors thank Edward Zipf, Rena SolbergKassel, Thomas Debski, Lawrence Young, Michael Beringer, and Ted Edwards for their assistance. Address all correspondence to Kenneth A. Perkins, Westem Psychiatric Institute and Clinic, 3811 O'Hara Street, Pittsburgh, PA 15213. may occur (Lipman, Blumenkopf, \& Parris, 1987). Two common means of thermal stimulation are radiant (light) heat and conducted heat (direct contact between stimulator and skin). A thorough review of radiant- and conductedheat-pain-stimulation methodology is beyond the scope of this report. However, several methods of conductedheat stimulation have recently been described that assess reliability of detection of small changes in intensity of thermal stimulation of cutaneous tissue in humans and animals (e.g., Gracely et al., 1988; Kenshalo et al., 1989). The objective of these methods is to determine psychophysical functions of heat-perception sensitivity. Lengthy and repeated exposure of subjects to thermal stimulation is required to derive these functions. There is little question of the elegance and reliability of these refined psychophysical techniques. Such procedures may not be feasible, however, for some research applications such as studies of acute effects of rapidly acting drugs. Most current methods of radiant-heat stimulation are derived from the pioneering work of Hardy et al. (1952), as well as the more recent work of Clark (e.g., Clark \& Yang, 1983) and others (see Chery-Croze, 1983). Highly precise methods of radiant-heat-pain stimulation are available, such as laser pulses (Arendt-Nielsen, Zachariae, \& Bjerring, 1990; Mor \& Carmon, 1975), but their expense may make them impractical for widespread use.

We have devised a relatively inexpensive but reliable device that assesses radiant-heat-pain threshold in healthy human subjects and that can be operated via IBM-compatible personal computer. This device was adapted from that of Hardy et al. (1952) and was designed to provide reliable values for pain threshold in nonclinical research with a minimum of subject preparation (e.g., training trials). Pain threshold rather than tolerance was examined 
because the shorter length of exposure and/or the lower intensity of the stimulation required to reach threshold is less anxiety provoking than that required to reach pain tolerance and makes tissue damage less likely. In this paper, we describe our method and present results from several studies that examine the reliability of earlier versions of the method and of the current procedure.

\section{GENERAL METHODS}

\section{Radiant-Heat-Pain-Threshold Assessment Device}

The radiant-heat-stimulus device is adapted from the "dolorimeter" of Hardy et al. (1952). Its operation is generally controlled by an AST Research Model 70 (IBM ATcompatible) personal computer. The radiant-heat source is a Kodak Kodaslide Master slide projector (Eastman Kodak) with a 1000 -W quartz tungsten-halogen projection lamp (Model BTR, General Electric). (The Kodaslide Master projector is an older model and may not be available new. However, other high-intensity projectors may be sufficient for this purpose.) The intensity of the light heat is controlled by a variable autotransformer (i.e., voltage regulator, range $=0-140 \mathrm{~V}$, Staco Energy Products) connected to the projector's light source. (The projector's fan must be provided with a separate connection to a power source to maintain cooling of the apparatus independent of voltage setting.) In most of our research, we have maintained the regulator at $75 \mathrm{~V}$, which allows for approximately $535 \mathrm{~mW}$ of heat from the $1000-\mathrm{W}$ bulb as determined from readings on a disk calorimeter (laser power meter, Scientech Model 36-0001). This intensity is low enough to minimize artifacts accompanying rapid rises in temperature (Yarnitsky \& Ochoa, 1990) and to prevent accidental tissue damage, but it is sufficient to produce pain threshold in normal subjects, generally within $60 \mathrm{sec}$. A high-temperature thermistor (Keystone Carbon) attached to a Wheatstone bridge and monitored by an $\mathrm{A} / \mathrm{D}$ convertor on the computer is used to monitor the relative intensity of the bulb over time and to determine the reliability of the heat output. The light is focused by a double-convex lens (6.4-cm diameter, Edmund Scientific) attached between the projector's collimating lens and the point of stimulation (i.e., subject's skin). The doubleconvex lens is located $2.5 \mathrm{~cm}$ from the collimating lens and $7 \mathrm{~cm}$ from the subject's skin. Precise onset and offset of light heat is controlled by an electronic shutter (Uniblitz Model 225L, Advanced Electromechanical Engineering) attached to the front of the projector, as shown in Figure 1.

The entire task is controlled by a program written in Microsoft QuickBASIC Version 4.5, which is available from the authors. When the experimenter initiates the task, the shutter opens at a random interval varying between 2 and $12 \mathrm{sec}$ (to prevent subjects from predicting the shutter opening). The shutter is controlled by a shutter driver (Uniblitz Model 100), which is signaled by com-

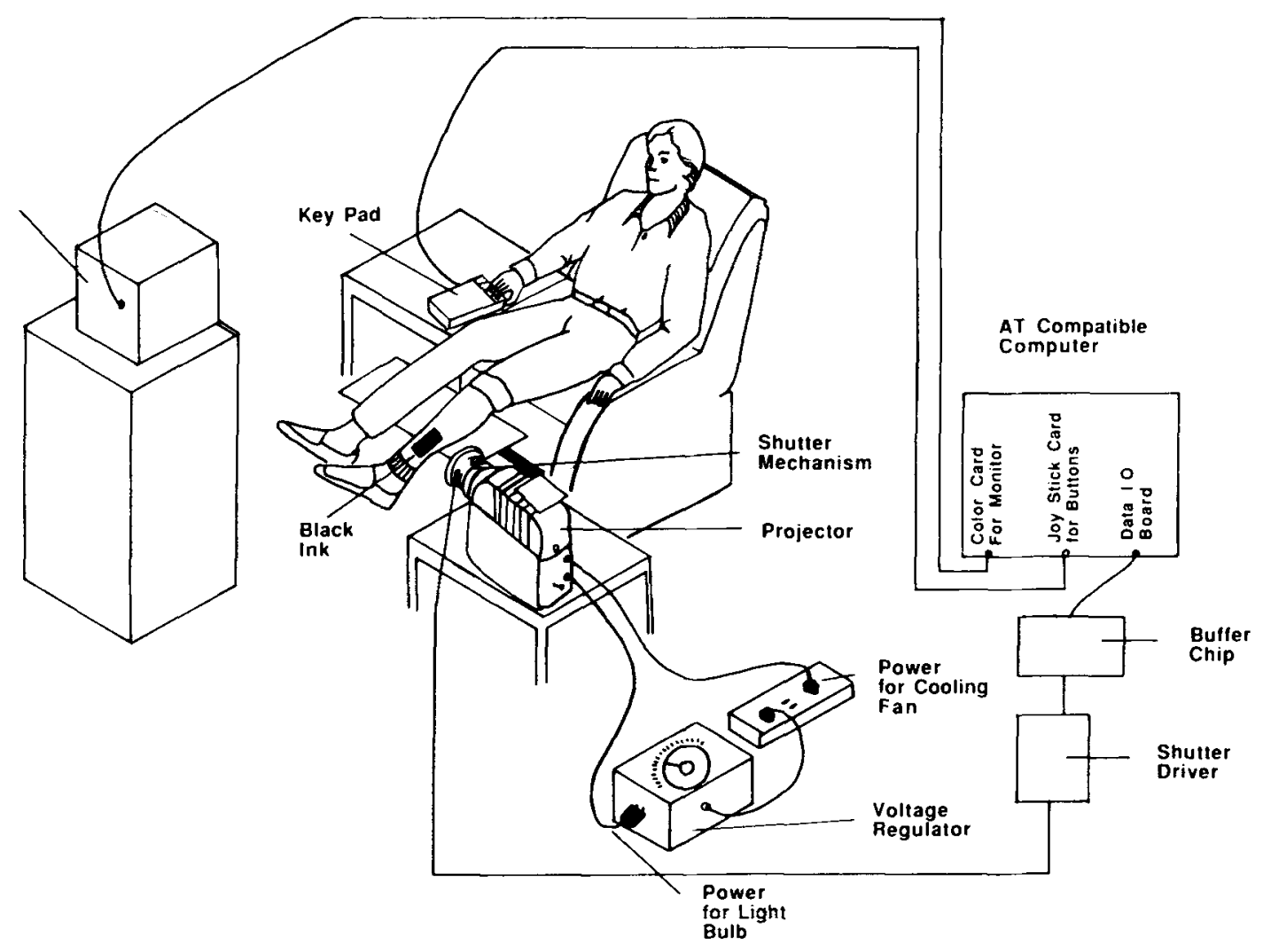

Figure 1. Thermal-stimulus apparatus. 
puter through data-output ports on a data-acquisition board (DASH-16, Metrabyte) or through any 5-V TTL signal available on standard output ports of IBM-compatible personal computers. A buffer chip (SN54LS/74LS244, Motorola) is used to protect the output ports on the computer from power surges. A four-button keypad (Apple), or any other response device, connected to a game card (Game Card III Plus, CH Products) on the computer records subject responses (see below). The computer's internal timer is used to record response latencies to the nearest $.01 \mathrm{sec}$. Approximate cost of these instruments is well under $\$ 1,000$, excluding computer and optional data-acquisition board.

\section{Subject Preparation and Instructions}

Throughout testing, the subject is seated in a comfortable reclining armchair within a sound-attenuated, climatecontrolled room. The light heat is applied to a $4-\mathrm{cm}^{2}$ area of the outside of the subject's leg, approximately $5 \mathrm{~cm}$ above the ankle (Figure 1). This area is prepared with an even coating of waterproof black India ink to standardize heat absorption across subjects' variations in skin pigmentation (Hardy et al., 1952). (Ink is removed by washing area with alcohol.) During an assessment trial, the subject's leg remains elevated on the footrest of the armchair and is placed directly against a 5-mm-thick rubber ring attached to the shutter around the opening. The rubber ring helps standardize distance between the double-convex focusing lens and the leg $(7 \mathrm{~cm})$ and prevents the leg from touching the shutter.

The subject is then introduced to the task by computerprovided instructions displayed on the video monitor approximately $2 \mathrm{~m}$ in front of him/her (see Figure 1), and he/she engages in a practice trial to familiarize him/herself with the task. After correct leg placement is verified, the task is initiated and the subject is shown a "sensation guide" on the computer screen that consists of a 4-point visual analog scale (VAS), with descriptors ranging left to right from "slight heat" to "warm" to "hot" to "pain." After brief presentation of all four descriptors of the scale, "slight heat" is highlighted on the monitor and the other descriptors are removed. The subject is instructed to press the first key on the keypad when he/she first detects any sensation of heat at all on his/her leg ("slight heat" = heat detection). At that point, "slight heat" is removed from the VAS on the screen and "warm" appears by itself, signaling the subject to press the second key when a "definite" sensation of warmth is felt. "Warm" is then removed from the VAS on the screen and "hot" appears, signaling the subject to press the third key when his/her leg begins to feel "hot" but not painful. Then "hot" is removed from the VAS and "pain" appears, signaling the subject to press the fourth (last) key on the keypad when the sensation changes from "hot" to a "prickly pain." These instructions are based on procedures used in past heat-pain research (Chery-Croze, 1983; Hardy et al., 1952). Laten- cies between shutter onset and the pressing of each of the four keys are recorded by computer. As soon as the subject presses the "pain" key, the computer activates the shutter driver to close the shutter, ending the task.

It is strongly emphasized to the subject that initial onset of pain is what is being assessed and not pain endurance (i.e., tolerance). Although the subject is told what types of sensations to expect, no information is given about expected latency to any of the sensations, which is the variable of interest. All four latencies are obtained by the computer, but only the last (pain threshold) is included in analyses. The earlier sensations ("slight" - heat detection, "warm," "hot") are employed only to keep the subject focused on the task and to "guide" his/her perception from heat detection to pain threshold. A sufficient interval of time is required between heat presentations to allow the skin temperature at the site of stimulation to cool and return to prestimulation temperature. We employed an interval of approximately $10 \mathrm{~min}$ in most of our research, but a shorter interval may be satisfactory. Intervals as short as 1 or 2 min may be acceptable if the site of stimulation is changed between heat presentations (Hardy et al., 1952), although this may also introduce greater variability in pain-threshold latencies.

\section{Overview of Reliability Studies}

Two studies and additional pilot research, presented below, were conducted to examine the reliability of this method of assessing the radiant-heat-pain threshold. In all research, the subjects provided informed consent to participate. Study 1 determined within-session reliability of each measure at each of three levels of heat stimulus using the palm of the hand as the site of heat stimulus. The palm was thought to be sensitive to variations in heat intensity and, possibly, to antinociceptive effects of drugs. As shown below, however, substantial variability was observed. Subsequent pilot research with smaller groups of subjects determined both within- and between-session reliability of measures using the palm or the calf of the left leg. The bulb used in this earlier research was a tungsten bulb (Model DFD, General Electric), which proved to be unreliable across periods of weeks, as noted below. Study 2, which examined within- and between-session reliability of pain threshold using the leg, employed the quartz tungsten-halogen bulb described above. This research is presented in chronological order and demonstrates factors that influence the reliability of the method.

Reliability of pain-detection threshold across trials was determined by coefficient of variation for each subject $[C V=(S D / M) \times 100]$ and presented as group mean $\pm S E C V$. Consistency of subjects' mean threshold across sessions (pilot work and Study 2) was also determined by $C V$. Between-subject differences in both latency to pain threshold and $C V$ of threshold due to heat intensity (Study 1) and gender (both studies) were analyzed by analysis of variance (ANOVA). 


\section{STUDIES OF RELIABILITY}

\section{Study 1. Within-Session Reliability at Different Heat Intensities}

Method. Twenty males $(M \pm S E$ age $=22.9 \pm .9$ years $)$ and 20 females (21.4 1.7 years) participated in one morning session. The subjects first had the centers of their left palms prepared with India ink, similar to the manner described above. Subsequently, the subjects were presented with three trials of the thermal stimulus. Each trial consisted of three exposures, one with the voltage regulator set at $65 \mathrm{~V}$ ("mild"), one at $70 \mathrm{~V}$ ("moderate"), and the third at $80 \mathrm{~V}$ ("high"). The three exposures within each trial period were separated by at least $5 \mathrm{~min}$, and one trial period occurred every $20 \mathrm{~min}$. Order of presentation of the three heat levels was counterbalanced across trials within subjects. Some subjects appeared to be insensitive to the mild heat level, and we employed a cutoff of $180 \mathrm{sec}$ for pain-threshold responses since it was assumed that the stimulus was insufficient to produce "prickly pain" in some subjects (see below). CVs were therefore not calculated for these subjects.

Results. As shown in Figure 2, latencies to pain threshold were distinct between voltage levels (i.e., heat intensities) but somewhat consistent across trials within each level. $M \pm S E C V$ s for heat-pain threshold were $14.4 \pm 1.8$, $15.3 \pm 1.4$, and $10.3 \pm 1.1$ for stimulation at 65,70 , and $80 \mathrm{~V}$, respectively $[F(2,48)=2.02, p>.10$ from ANOVA of $C V \mathrm{~s}$ ]. There was no significant gender difference in $C V(F<1)$. The ANOVA of threshold latencies indicated a highly significant difference between heat intensities $[F(2,70)=45.72, p<.001]$, as expected, but also a significant trials effect $[F(2,70)=4.39, p<.05]$, despite the apparent consistency across stimulus presentations. There were no significant effects involving gender $(F \mathrm{~s}<1)$. Despite moderate within-subject reliability of latencies, there was extensive between-subject variability, as the range in pain-threshold latency was 15.8 to $180 \mathrm{sec}$ (cutoff, reached by 14 subjects on at least one trial) for the mild level, 7.2 to $180 \mathrm{sec}$ (reached by 7 subjects) for moderate heat, and 4.1 to $46.6 \mathrm{sec}$ for high-intensity heat. Furthermore, males were slightly, although not significantly, more likely to reach the cutoff of $180 \mathrm{sec}$ during at least one trial ( 9 of 20 males vs. 5 of 20 females, $\chi^{2}=1.76, p>.10$ ), suggesting that elimination of the cutoff might have revealed a significantly longer latency to pain threshold in males. This possibility was further explored in Study 2 (see below). At the end of the session, subjects completed a VAS item that asked, "How much does it bother you to experience pain like that involved in this study?" On a 10-point scale anchored by "not at all" (0) to "extremely" (10), with "moderately" at the midpoint (5), $M \pm S E$ response was $1.2 \pm 0.2$, suggesting that pain experienced by this method is not anxiety producing, as expected.

Discussion. The significant trials effect in the first study indicated significant variability across trials, which appeared greater at lower heat intensities. This finding is consistent with past observations that reliability is poorer, perhaps due to response bias, if stimulation is substantially below pain threshold or latency to threshold is long (e.g., Chapman et al., 1985). In addition, the fact that a number of subjects reached the cutoff of $180 \mathrm{sec}$ following exposure at 65 and $70 \mathrm{~V}$ suggested that these in-

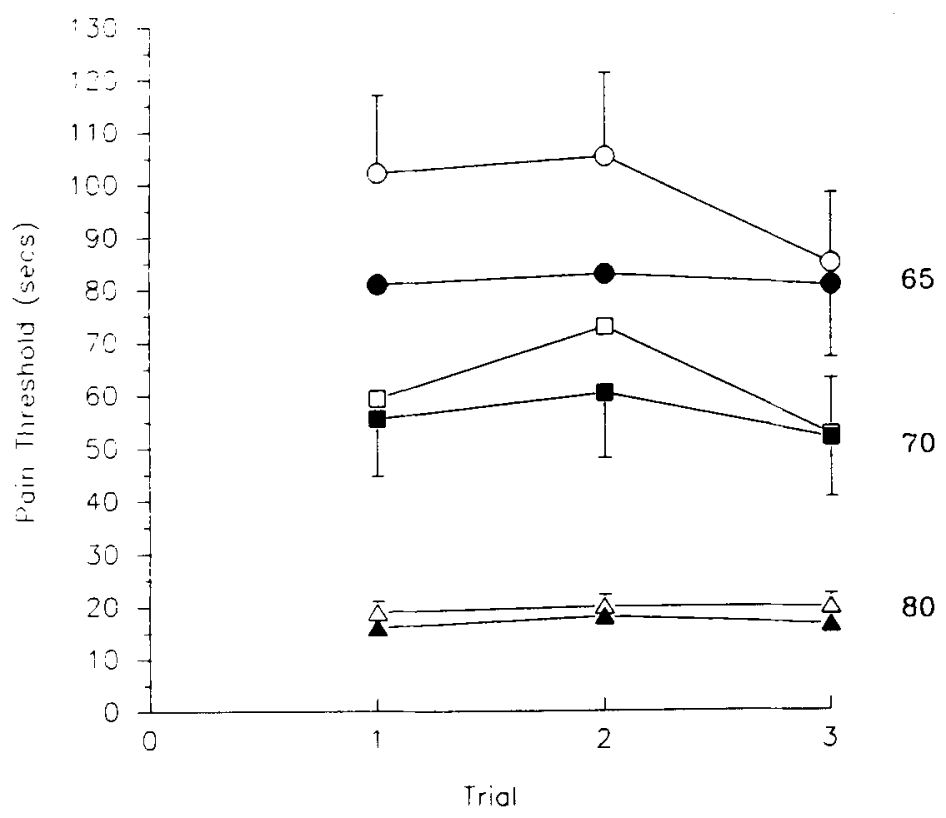

Figure 2. Mean $\pm S E$ pain-threshold latencies for 20 males (open symbols) and 20 females (filled symbois) across three trials at each of three voltage levels (i.e., heat intensities: mild $-65 \mathrm{~V}$; moderate $-70 \mathrm{~V}$; high $-80 \mathrm{~V}$ ) during a single session using the paim as the site of heat stimulation. 
tensities were insufficient to produce pain in some subjects. Thus, in subsequent examinations of the within- and between-session reliability of this procedure, we chose a single, moderately high intensity level $(75$ or $80 \mathrm{~V})$ because of the improved reliability (see results for $80 \mathrm{~V}$ in Figure 2).

\section{Additional Pilot Work}

Subsequent pilot work was performed to test reliability across days as well as trials. In a test using exposure at $75 \mathrm{~V}$ and the palm as the site of stimulation, results for 2 males and 1 female participating in three trials on each of three different days revealed moderate reliability in threshold across trials within sessions (mean $C V=$ $11.1 \pm 4.2)$ and across mean session latencies $(C V=$ $10.9 \pm 2.9$ ). Nevertheless, thresholds tended to increase between sessions $(M \mathrm{~s}=26.5,27.1$, and $34.4 \mathrm{sec}$ across Days 1-3, respectively, averaged across trials) and across trials within sessions $(M \mathrm{~s}=25.6,28.2$, and $34.1 \mathrm{sec}$ across trials 1-3, 'respectively, averaged across days). Although no formal follow-up evaluation was done to determine the exact cause of the observed variability within sessions, it is possible that it was due to changes in palm sweat activity. Therefore, the outside of the calf of the left leg (the procedure described in General Methods, above) was used in subsequent studies (see also Hardy et al., 1952). In the next test, 4 male subjects participated in three morning sessions, held 3-5 days apart, that involved stimulation of the calf of the left leg with exposure at $80 \mathrm{~V}$. Results indicated substantially improved reliability in pain thresholds across trials within sessions, as evidenced by lower $C V s(M=6.4 \pm 1.3)$. Mean latencies were $15.2,15.6$, and $14.8 \mathrm{sec}$ across trials $1-3$, respectively, averaged across days. However, reliability between sessions was poorer $(C V=15.9 \pm 6.4)$, as latencies increased gradually across sessions $(M s=11.7,15.5$, and $18.4 \mathrm{sec}$ for Days 1-3, respectively, averaged across trials).

Information on the performance of light bulbs indicated that the tungsten bulbs normally used with this projector show a linear decrease in light intensity over time, as the tungsten filament thins and tungsten is deposited on the side of the bulb (Oriel Co., 1989). This results in a reduction of output within the infrared range. Such a decay in intensity could explain the general tendency for latencies to increase across sessions in the pilot research, which was carried out over a 2-month period. To overcome this problem, we employed a quartz tungsten-halogen bulb, as described in General Methods. This bulb shows a very slow decline in intensity over time (Oriel Co., 1989). Use of the thermistor, noted in General Methods, allows for monitoring of the bulb's performance and can indicate when a replacement is needed.

\section{Study 2. Within- and Between-Session Reliability}

Method. Subjects were 11 males ( $22.5 \pm 1.0$ years) and 14 females $(22.3 \pm 0.6$ years) who participated in three morning sessions held 6-35 days apart (median $=14$ ). This study took place over an 8-month period. Procedures for this study were identical to those of the previous one except for the use of the tungsten-halogen bulb and exposure at $75 \mathrm{~V}$ (same as pilot research with the palm, above). After instructions and a practice trial, the subjects were exposed to three trials with the stimulus, each separated by $10 \mathrm{~min}$. (The first session for $1 \mathrm{male}$ was repeated because of unexplained excessively long latenciesall greater than $80 \mathrm{sec}$-which were not observed in subsequent sessions.)

Results. As shown in Figure 3, within-session reliability remained excellent with this procedure, while betweensession reliability improved somewhat. Mean $\mathrm{CVs}$ in

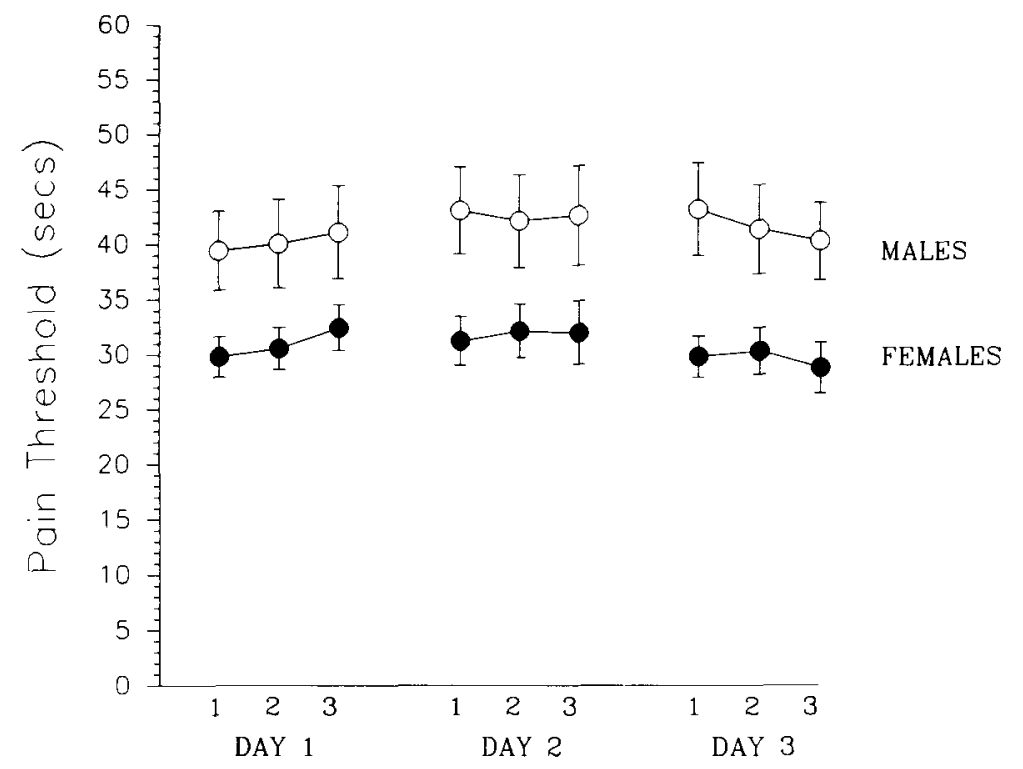

Figure 3. Mean $\pm S E$ pain-threshold latencies for 11 males and 14 females across three trials within each of three sessions using the left calf (intensity $=75 \mathrm{~V}$ ). 
threshold latencies across trials for Days 1-3 (i.e., withinsession reliability) were $9.1 \pm 1.6,7.6 \pm 1.0$, and $7.2 \pm 1.2$, respectively. $C V$ across mean session latencies (i.e., between-session reliability) was $14.2 \pm 1.4$, and there was no systematic change in latencies across sessions $(M \mathrm{~s}=$ 35.1, 36.6, and 35.0 sec for Days 1-3, respectively, averaged across trials). The ANOVA of $C V$ values revealed no significant effects of gender $[F(1,23)=1.71, p>.10]$, day $(F<1)$, or gender $\times$ day interaction $[F(2,46)=$ $2.19, p>.10$ ]. However, ANOVA of pain-threshold latencies revealed a highly significant gender difference $[F(1,23)=9.98, p<.005]$, with shorter latencies among females as compared with males, as shown in Figure 3. There were no significant main or interaction effects of days or trials and no interaction effects involving gender on pain-threshold latency (all $F \mathbf{s}<1$ ). Since there was substantial variability across subjects in the number of days between sessions, the correlation between $C V$ for session means (between-session reliability) and number of days to complete the three sessions was obtained. Results showed a nonsignificant Pearson correlation of .02 , further indicating that reliability of pain threshold with this method was unaffected by passage of time between assessments.

\section{GENERAL DISCUSSION}

The method described here appears to provide reliable assessment, with minimal expense and subject preparation, of heat-pain threshold in healthy, nonclinical subjects. Results from the first study and subsequent pilot work indicate that several easily correctable procedural and technical variables may influence reliability of pain thresholds. In Study 1, it was observed that small changes in voltage (e.g., 65 vs. $70 \mathrm{~V}$ ) can produce substantial differences in latencies, requiring precision in the setting of the voltage, and that lower intensities (producing longer latencies) are associated with poorer reliability. In followup pilot work, latencies increased across trials within sessions, suggesting that the palm may be inadequate for use in studies of acute changes in heat-pain threshold. When the calf was used as the site of heat stimulation, withinsession reliability was greatly improved but betweensession variability was still somewhat poor, most likely due to decay over time in heat output by the standard tungsten bulbs. Finally, in Study 2, use of the quartz tungstenhalogen bulb with the calf demonstrated excellent withinsession and good between-session reliability.

Although little subject preparation is required, some care needs to be taken to ensure accurate, reliable values. Ambient temperature must be maintained to prevent sweating and changes in skin temperature, which can influence latencies to pain threshold. Ideally, skin temperature at the stimulation site should be monitored with a temperature probe to ensure a stable skin temperature prior to each exposure. Preparation of the leg with India ink must be consistent between subjects and across sessions within subjects (Hardy et al., 1952), and leg place- ment next to the shutter must be monitored during heat exposure. Finally, as indicated, bulb performance should be evaluated on a regular basis (e.g., every week) with a high-temperature thermistor or other device that provides an indication of the bulb's output.

A possible limitation of the device as described here is the lack of monitoring of absolute energy intensities produced by the radiant-heat source, which may be necessary to ensure precisely equal stimulation between different subjects and between sessions with the same subject. This limitation might have little impact on results of acute within-session manipulations but could complicate comparisons between subjects tested on different days. Devices to calibrate energy intensity are available (e.g., radiometer or disk calorimeter), although adding such a device would easily triple the total cost of this method. For the purposes for which this method is intended, we believe use of an infrared thermometer (Omega Engineering) or the thermistor used here provide an adequate gauge of relative changes in intensity to ensure some consistency in intensity across presentations.

It may be possible to use this device without the aid of a computer, thereby reducing the cost even further. Instructions could be provided by tape recorder or in person, the thermal stimulus onset and offset could be activated manually, and time to an oral response of "pain" could be obtained by stopwatch, though with some loss of precision. However, accomplishing these functions with the computer not only provides greater precision of measurement but allows for automatic recording and storage of data and integration of this task within a battery of other subjective or behavioral tasks adaptable for the computer (e.g., Snyder \& Henningfield, 1989). The widespread availability of these computers also makes this integration easily attainable for most researchers.

There may be a number of applications of this method to basic behavioral research with healthy human populations. Because reliability within sessions tended to be better than reliability between sessions, this method may be sensitive to acute behavioral or pharmacological manipulations within sessions, although the specific mechanisms responsible for changes in response latencies (e.g., true antinociception vs. suppression of motor responding) need to be carefully considered (Chapman et al., 1985). Several commonly used drugs may alter pain sensitivity, possibly a critical determinant of their abuse-liability potential. For example, nicotine has been widely shown to produce antinociception in animals (Tripathi, Martin, \& Aceto, 1982), but studies with humans have provided inconsistent results (e.g., Pomerleau et al., 1984; Waller et al., 1983). Using this procedure, we have recently found that nicotine intake may lead to increases in radiantheat-pain threshold of smokers and nonsmokers (Perkins et al., in press). Comparison of drug effects on antinociception using this method with effects on other subjective or behavioral responses may provide an indication of the degree to which the antinociceptive effects relate to other reinforcing effects of drugs. In addition, 
comparison of results with this method and effects using other methods (e.g., cold pressor) may determine generalizability of radiant-heat-pain threshold with other indices of pain threshold (Harris \& Rollman, 1983). Similarly, the effects of instructional set or distraction, which may be substantial with pain-detection methods such as cold pressor (e.g., Hodes, Howland, Lightfoot, \& Cleeland, 1990), could be compared between radiant heat using this method and the other procedures. Manipulation of higher heat intensities using the voltage regulator, such as via magnitude estimation procedures, could also allow brief, fixed exposures to estimate psychophysical pain functions, although other, more precise methods for this purpose have been developed (Chapman et al., 1985). Finally, as evidenced by the significant gender difference in pain threshold found in Study 2, this method may be sensitive to individual differences in pain threshold associated with various stable characteristics of interest. However, as noted earlier, the lack of absolute energy intensities available with the method as presented here could limit its use in between-subject comparisons.

\section{REFERENCES}

Arendt-Nielsen, L., Zachariae, R., Bierring, P. (1990). Quantitative evaluation of hypnotically suggested hyperaesthesia and analgesia by painful laser stimulation. Pain, 42, 243-251.

BEECHER, H. K. (1959). Measurement of subjective responses: Quantitative effects of drugs. New York: Oxford University Press.

Chapman, C. R., Casey, K. L., Dubner, R., Foley, K. M., Gracely, R. H., Reading, A. E. (1985). Pain measurement: An overview. Pain, 22, 1-31.

Chen, A. C. N., Dworkin, S. F., Haug, J., Gehrig, J. (1989). Human pain responsivity in a tonic pain model: Psychological determinants. Pain, 37, 143-160.

Chery-Croze, S. (1983). Painful sensation induced by thermal cutaneous stimulus. Pain, 17, 109-137.

Clark, W. C., Yang, J. C. (1983). Applications of sensory decision theory to problems in laboratory and clinical pain. In R. Melzack (Ed.), Pain measurement and assessment (pp. 15-25). New York: Raven Press.

Collier, J. L., Merta, B. M., \& Spear, F. G. (1990). Experimental changes in pain threshold and severe pain threshold for electrically induced pain. Journal of Psychosomatic Research, 34, 99-102.
Gracely, R. H., Lota, L., Walter, D. J., Dubner, R. (1988). A multiple random staircase method of psychophysical pain assessment. Pain, 32, 55-63.

HANDWERKER, H. O. (1984). Some aspects of pain measurement in man. Drug Research, 34, 1093-1095.

HaRdY, J. D., WolfF, H. G., GoOdell, H. (1952). Pain sensation and reaction. Baltimore: Williams \& Wilkins.

Harris, G., Rollman, G. B. (1983). The validity of experimental pain measures. Pain, 17, 369-376.

Hodes, R. L., Howland, E. W., Lightfoot, N., \& Cleeland, C. S. (1990). The effects of distraction on responses to cold pressor pain. Pain, 41, 109-114.

Kenshalo, D. R., Anton, F., Dubner, R. (1989). The detection and perceived intensity of noxious thermal stimuli in monkey and in human. Journal of Neurophysiology, 62, 429-436.

Lipman, J. J., Blumenkopf, B., \& Parris, W. C. V. (1987). Chronic pain assessment using heat beam dolorimetry. Pain, 30, 59-67.

MOR, J., \& CARMON, A. (1975). Laser emitted radiant heat for pain research. Pain, 1, 233-237.

ORIEL Co. (1989). Light sources, monochromators, and detection systems, Vol. 2 (product catalog). (Available from Oriel Co., P.O. Box 872, Stratford, CT 06497)

Perkins, K. A., Grobe, J. E., Epstein, L. H., Stiller, R. L., SolberG-Kassel, R. D., \& JACOB, R. G. (in press). Chronic tolerance to cardiovascular, subjective, and behavioral effects of nicotine in humans. In Problems of Drug Dependence 1991 (Proceedings of the 53rd Annual Scientific Meeting; Committee on Problems of Drug Dependence, NIDA research monograph). Rockville, MD: National Institute on Drug Abuse.

Pomerleau, O. F., Turk, D., * Fertig, J. (1984). The effects of cigarette smoking on pain and anxiety. Addictive Behaviors, 9. 256-271.

Seltzer, C., Friedman, G., Siggelaub, A., Collen, M. (1974). Smoking habits and pain tolerance. Archives of Environmental Health, 29, 170-172.

SNYDER, F. R. , HeNNINGFELD, J. E. (1989). Effects of nicotine administration following $12 \mathrm{~h}$ of tobacco deprivation: Assessment on computerized performance tasks. Psychopharmacology, 97, 17-22.

Tripathi, H., MARTIN, B., ACETo, M. (1982). Nicotine-induced antinociception in rats and mice: Correlation with nicotine brain levels. Journal of Pharmacology \& Experimental Therapeutics, 22, 91-96.

Waller, D., Schalling, D., Levander, S., Edman, G. (1983). Smoking, pain tolerance, and physiological activation. Psychopharmacology, 79, 193-198.

YARNITSKY, D., \& ОСноА, J. L. (1990). Studies of heat pain sensation in man: Perception thresholds, rate of stimulus rise and reaction time. Pain, 40, 85-91.

(Manuscript received November 29, 1990; revision accepted for publication November 5, 1991.) 